\title{
Path Loss Prediction Using Fuzzy Inference System and Ellipsoidal Rules
}

\author{
${ }^{1}$ Mario Versaci, ${ }^{1}$ Salvatore Calcagno, ${ }^{1}$ Fabio La Foresta and ${ }^{2}$ Biagio Cammaroto \\ ${ }^{1}$ Department of MECMAT, University Mediterranea, Reggio Calabria, Italy \\ ${ }^{2}$ Department of Civil Engineering, University of Messina, Contrada di Dio, I-98166 Messina, Italy
}

Received 2012-09-15, Revised 2012-09-16; Accepted 2012-12-06

\begin{abstract}
It is well known as the prediction of radio wave path loss in urban environment plays a key role in order to correctly plan wireless systems and mobile communication networks. To obtain more flexible prediction models able to give accurate results, in recent years Soft computing Techniques has been exploited. In this study, a novel approach based on ellipsoidal fuzzy inference system EFIS is investigated. Results compared with those provided by the Okumura Hata model and the standard Fuzzy Inference System approach (FIS) show superior performances of the EFIS approach.
\end{abstract}

Keywords: Path Loss, Soft Computing, Fuzzy Inference System, Ellipsoidal Rules

\section{INTRODUCTION}

The prediction of the path loss in an urban environment is of paramount importance for the design and planning of the modern mobile communication systems. As a matter of fact, information on the behavior of this parameter is crucial in determining link budgets, cell sizes and reuse distances. At this purpose, empirical and theoretical models for the path loss prediction have been developed during the years. However, both the class of models suffered of some drawbacks. Classical empirical models are often unsatisfactory in term of prediction accuracy and rarely they adapt well to different types of propagation environment, while theoretical models are lacking of computational efficiency. To obtain more flexible empirical models capable to give accurate prediction results, in recent years Soft Computing Techniques have been exploited (Neskovic et al., 2000). Soft computing techniques (artificial neural networks, genetic algorithms, fuzzy logic models, particle swarm techniques) are an attractive alternatives to the standard, well established hard computing paradigms and they have been exploited with success to solve a wide variety of problems such as modeling clustering of entropy topography in epileptic electroencephalography (Mammone et al., 2011a; 2011b; Labate et al., 2011a; 2011b; Mammone et al., 2012; Morabito et al., 2012), dynamical reconstruction of road longitudinal profiles (Costantino et al., 2012), prediction problems in nuclear fusion applications (Versaci and Morabito, 2003), for modeling microwave devices and antennas (Angiulli and Versaci, 2002; 2003). In particular, among the different methodological approaches provided by Soft Computing, in this study, a novel approach based on the fuzzy inference system EFIS coupled with ellipsoidal rules to forecast the path loss behavior, is investigated. Actually, in the literature the ellipsoidal fuzzy systems have not much been used because are characterized by means of geometrical complexity. So, their exploitation is often limited to applications where a better precision is required. The study is organized as follows: The next section recalls basics on the path loss concept and the Okumura-Hata model. Then, an overview the EFIS fuzzy approach is given. Finally numerical results and conclusions are drawn.

Corresponding Author: Mario Versaci, Department MECMAT, University Mediterranea, Reggio Calabria, Italy 


\section{MATERIALS AND METHODS}

\subsection{Path Loss and the Okumura-Hata Model}

Path Loss L is a measure of the signal level attenuation caused by propagation mechanisms. It is defined as Eq. 1:

$$
\mathrm{L}_{\mathrm{dB}}=10 \log \left(\frac{\mathrm{P}_{\mathrm{t}}}{\mathrm{P}_{\mathrm{r}}}\right)
$$

where, $\mathrm{P}_{\mathrm{t}}$ and $\mathrm{P}_{\mathrm{r}}$ are the transmitted and received power, respectively. The Okumura-Hata model is likely to be the most used and widespread path loss prediction model. It is based on experimental data collected in Japan at different frequencies including in the range 150$1920 \mathrm{MHz}$, for a height of the transmitting antenna ranging between $30-1000$ meters. It gives the average path loss for urban areas as Eq. 2:

$$
\begin{aligned}
\overline{\mathrm{L}}_{\mathrm{UA}, \mathrm{dB}} & =69.55+26.16 \log \left(\mathrm{f}_{\mathrm{c}}\right) \\
& -13.82 \log \left(\mathrm{h}_{\mathrm{eff}}\right)+-\mathrm{a}\left(\mathrm{h}_{\mathrm{rx}}\right)+\Delta \log (\mathrm{d})
\end{aligned}
$$

Where:

$$
\begin{aligned}
& \begin{aligned}
& \mathrm{f}_{\mathrm{c}}= \text { The carrier frequency in } \mathrm{MHz} \\
& \mathrm{d}= \text { The antenna separation distance (ranging } \\
&\text { between } 1-20 \mathrm{Km}) \\
& \mathrm{h}_{\mathrm{eff}}= \text { The effective height of the transmitting } \\
& \text { antenna } \\
& \mathrm{h}_{\mathrm{rx}}= \text { The height of the receiving antenna (ranging } \\
&\text { between } 1-10 \mathrm{~m}) \\
& \mathrm{a}\left(\mathrm{h}_{\mathrm{rx}}\right)= \text { The correction factor }(\mathrm{dB}) \text { and } \Delta \text { is Eq. } 3 \text { : } \\
& \Delta=44.9-6.55 \log \left(\mathrm{h}_{\text {eff }}\right)
\end{aligned}
\end{aligned}
$$

This model also assumes that there are no dominant obstacles between the transmitting and receiving antennas and that the ground profile changes slowly. The average path loss for suburban area is formulated as Eq. 4:

$$
\overline{\mathrm{L}}_{\mathrm{SUA}, \mathrm{dB}}=\overline{\mathrm{L}}_{\mathrm{UA}, \mathrm{dB}}-2\left(\log \left(\frac{\mathrm{f}_{\mathrm{c}}}{28}\right)\right)^{2}-5.4
$$

while in the rural open environment assumes the form Eq. 5:

$$
\begin{aligned}
\overline{\mathrm{L}}_{\mathrm{ROE}, \mathrm{dB}} & =\overline{\mathrm{L}}_{\mathrm{UA}, \mathrm{dB}}-4.78\left(\log \left(\mathrm{f}_{\mathrm{c}}\right)\right)^{2}+ \\
& +18.33\left(\log \left(\mathrm{f}_{\mathrm{c}}\right)\right)-40.49
\end{aligned}
$$

\subsection{Fuzzy Inference System and Ellipsoidal Rules: A Brief Review}

NFIS approach is a logical tool that maps inputs to outputs by bank of fuzzy rules providing a basic from which it is possible to take important decisions. Typically, a Sugeno-FIS can be structured into three sections: Membership functions, fuzzy operators and bank of if then rules which represents the inference of the procedure. Sugeno inference performs by the four following sections.

\subsection{Fuzzification of the Inputs}

It is imperative to determine the degree to which inputs (represented by fuzzy sets in Sugeno's inference) belong to each fuzzy set by means of membership functions. This degree represents the degree to which each if-part of each rules has been satisfied.

\subsection{Application of Fuzzy Connectives}

If the antecedent of a rule has more than one part, the fuzzy operator is applied to obtain one number representing the results of the antecedent for that rule. Using AND connective, the antecedent of each rules is active to the minimum values.

\subsection{Implication Procedure}

The consequent part of each rules, that Sugeno's inference structures by singletons, is activated to the results of the antecedent for that rules.

\subsection{Defuzzification}

The output is computed by a linear combination of the single fuzzy singletons active as reported to previous step. Nevertheless, to generate optimal FIS it is possible to exploit GENFIS $\AA$ procedure that exploits fuzzy subtractive clustering to determine the number of rules and antecedent membership functions and then uses linear least squares estimation to determine each rule's consequent equations (Jang, 1993). Regarding EFIS, the covariance of date identify ellipsoidal rules (patches). In particular, each rule is represented by an ellipsoid covering a portion of inputs-outputs space and they overlap. Geometrically, an ellipsoid $\mathrm{z}$ can be represented by eigenvectors and Eigen values of a positive definite matrix $A$ : If $n$ and $p$ are is the number of inputs and outputs respectively, $q=n+p$ represent the ellipsoid dimension Eq. 6:

$$
\begin{aligned}
\alpha^{2} & =(z-c)^{\mathrm{T}} \mathrm{A}(\mathrm{z}-\mathrm{c}) \\
& =(\mathrm{z}-\mathrm{c})^{\mathrm{T}} \mathrm{P} \Lambda \mathrm{P}^{\mathrm{T}}(\mathrm{z}-\mathrm{c})
\end{aligned}
$$


Where:

$\alpha=\in \mathrm{R}^{+}$

$\mathrm{c}=$ Centre of ellipsoid

$\mathrm{A}=$ Diagonal matrix of Eigen values of A

$\mathrm{P}=$ Orthogonal matrix that orient the ellipsoid

Its columns are the unitary eigenvectors while the lengths of semi axis are computed by Eq. 7:

$\frac{\alpha}{\sqrt{\lambda_{1}}}, \ldots, \frac{\alpha}{\sqrt{\lambda_{\mathrm{q}}}}$

Where:

$\lambda_{\mathrm{k}}=$ Eigen values of $\mathrm{A}$

$\mathrm{c}=$ Centre of ellipsoid

Finally, each ellipsoid represents a fuzzy rule and then, its projection on each possible values axis represents the support of membership function. In our case, triangular membership functions are considered (Dickerson and Kosko, 1996).

\section{RESULTS AND DISCUSSION}

In order to face the path loss prediction problem by means of the EFIS approach, we have considered an EFIS network having the following inputs: (i) the carrier frequency, (ii) the transmitting antenna gain, (iii) the receiving antenna gain, (iv) the distance from the radio- base station, (v) the height of the building (vi) the length of the street, (vii) the building separation, (viii) the height of the transmitting antenna, (ix) the height of the receiving antenna, while the output is the path loss value. For the designing and training our NFIS model, we have exploited the MatLab ${ }^{\circledR}$ Genfis toolbox (Jang, 1993). A database of 2000 path loss values has been built exploited a synthetic urban scenario model using Wireless InSite ${ }^{\circledR}$, a commercial software based on the ray-tracing method. Afterwards, a testing dataset of further 1000 path loss values has been built for testing the prediction ability of the model. Table 1 shows some parameter used in simulations. All computations have been carried out using a PC with two Intel Core2 CPUs at $1.66 \mathrm{GHz}$ and $4 \mathrm{~GB}$ of RAM. The performances of the EFIS network are compared with those offered by the Okumura Hata model and by a standard Fuzzy Inference System (FIS) network. The forecasting accuracy of these models have been evaluated in term of Root Mean Square Error (RMSE) and Mean Absolute Percentage Error (MAPE).

Figure 1 shows a comparison among the ray tracing value and the estimated path loss values as a function of the distance for the considered Okumura Hata, NFIS and EFIS models. It can be observed as, in the considered case, EFIS and NFIS networks give more better results than the Okumura Hata model, describing in a more faithful way the path loss trend.

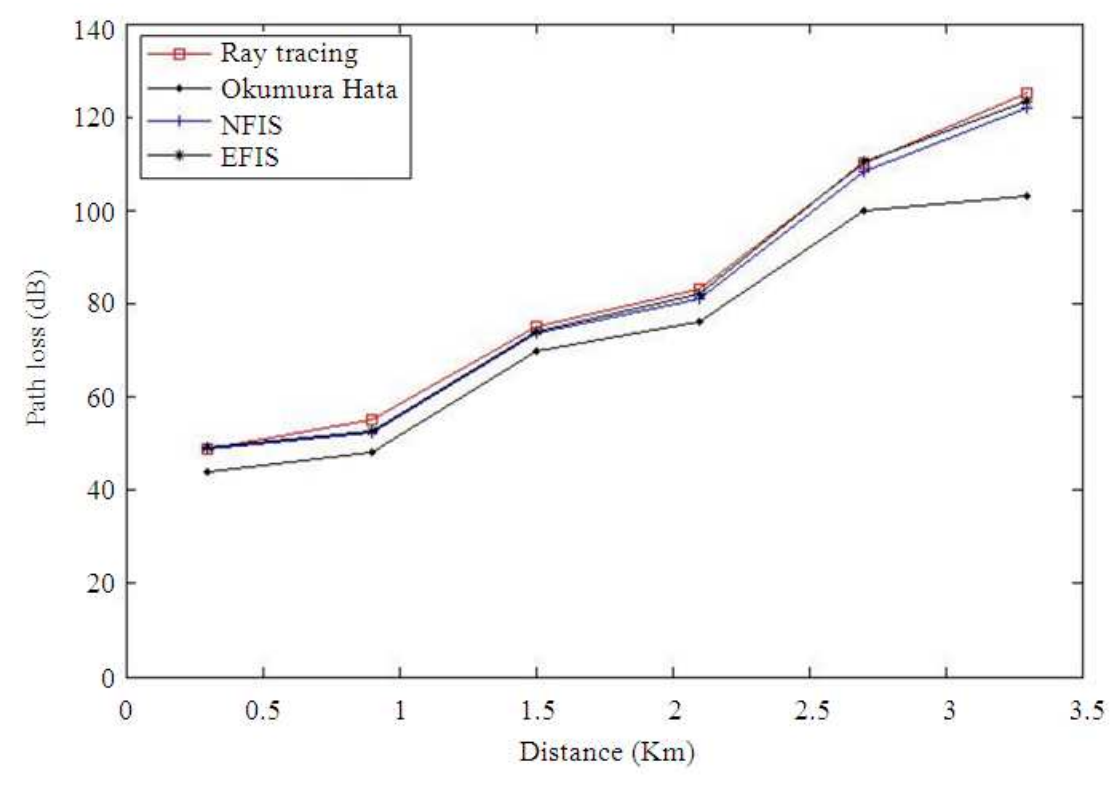

Fig. 1. Ray Tracing, NFIS, EFIS and Okumura-Hata path loss values versus distance 
Table 1. Wireless Insite parameters

\begin{tabular}{ll}
\hline Parameter & Range \\
\hline Carrier frequency & $900[\mathrm{MHz}]$ \\
Transmitting antenna gain & $16[\mathrm{dBi}]$ \\
Transmitting antenna height & $30 \mathrm{~m}$ \\
Receiving antenna height & $1.5 \mathrm{~m}$ \\
Range of the building heights & $12 \div 27 \mathrm{~m}$ \\
Buildings material & Concrete: $\varepsilon_{\mathrm{r}}=15$, \\
& $\sigma=0.015[\mathrm{~S} / \mathrm{m}]$ \\
\hline
\end{tabular}

Table 2. Root mean square error and means absolute percentage error

\begin{tabular}{lll}
\hline Method & RMSE & MAPE \\
\hline NFIS & 1.716 & 0.833 \\
EFIS & 0.833 & 1.716 \\
Okumura Hata & 9.330 & 9.333 \\
\hline
\end{tabular}

Table 3. NFIS and EFIS networks: Number of rules

\begin{tabular}{ll}
\hline Method & Number of rules \\
\hline NFIS & 21 \\
EFIS & 12 \\
\hline
\end{tabular}

In Table 2 are reported the performances of the considered models in term of RMSE and MAPE. It can be said that both NFIS and EFIS network show a good performance in terms of estimation. Nevertheless, referring to the number of rules (Table 3), the best performance is provided by EFIS network because, by means of 29 ellipsoidal rules, is able to evaluate the path loss with a RMSE and a MAPE smaller than $2 \%$.

\section{CONCLUSION}

In this study, a novel method for the path loss prediction Exploiting the Fuzzy Inference System in conjunction with ellipsoidal rules (EFIS) is presented. Results compared with those provided by the Okumura Hata model and the standard Fuzzy Inference System Approach (FIS) show superior performances of the proposed approach.

\section{REFERENCES}

1. Angiulli, G. and M. Versaci, 2002. A neurofuzzy network for the design of circular and triangular equilateral microstrip antennas. Int. J. Infrared Millimeter Waves, 23: 1513-1520. DOI: 10.1023/A:1020333704205
2. Angiulli, G. and M. Versaci, 2003. Resonant frequency evaluation of microstrip antennas using a neural-fuzzy approach. IEEE Trans. Mag., 39: 1333-1336. DOI: 10.1109/TMAG.2003.810172

3. Costantino, D., F.C. Morabito, F.G. Pratico and M. Versaci, 2012. Dynamical reconstruction of road longitudinal profiles: A theoretical and experimental study. Int. J. Model. Simul. Actapress, 32: 149-156. DOI: 10.2316/Journal.205.2012.3.205-4752 http://www.recurrenceplot.tk/bibliography.php?label=costantino2012

4. Dickerson, J.A. and B. Kosko, 1996. Fuzzy function approximation with ellipsoidal rules. IEEE Trans. Syst. Man Cybernetics-Part B: Cybernetics, 26: 542-560. DOI: 10.1109/3477.517030

5. Jang, J.S.R., 1993. ANFIS: Adaptive-networkbased fuzzy inference system. IEEE Trans. Syst. Man Cybernetics, 23: 665-685. DOI: 10.1109/21.256541

6. Labate, D., F.L. Foresta, G. Inuso and F.C. Morabito, 2011a. Remarks about wavelet analysis in the EEG artifacts detection. Proceedings of the 20th Italian Workshop on Neural Nets, (NN' 11), IOS Press Amsterdam, Netherlands, pp: 99-106. http://dl.acm.org/citation.cfm?id=1940646

7. Labate, D., F.L. Foresta, G. Inuso and F.C. Morabito, 2011b. Multiscale entropy analysis of artifactual EEG recordings. Frontiers Artif. Intell. Appli., 234: 170-177. DOI: 10.3233/9781-60750-972-1-170

8. Mammone, N., G. Inuso, F.L. Foresta, M. Versaci and F.C. Morabito, 2011a. Clustering of entropy topography in epileptic electroencephalography. Neural Comput. Appli., 20: 825-933. DOI: 10.1007/s00521-0100505-2

9. Mammone, N., F.L. Foresta and F.C. Morabito, $2011 \mathrm{~b}$. Discovering network phenomena in the epileptic electroencephalography through permutation entropy mapping. Proceedings of the 20th Italian Workshop on Neural Nets, (NN' 11), IOS Press Amsterdam, Netherlands, pp: 260-269. http://dl.acm.org/citation.cfm?id=1940666 
10. Mammone, N., F.L. Foresta and F.C. Morabito, 2012. Automatic artifact rejection from multichannel scalp EEG by wavelet ICA. IEEE Sensors J., 12: 533-542. DOI: 10.1109/JSEN.2011.2115236

11. Morabito, F.C., D. Labate, F.L. Foresta, A. Bramanti and G. Morabito et al., 2012. Multivariate multi-scale permutation entropy for complexity analysis of Alzheimer's disease EEG. Entropy, 14: 1186-1202. DOI: 10.3390/e 14071186

12. Neskovic, A., N. Neskovic and D. Paumovic, 2000. Indoor electric field level prediction model based on the artificial neural networks. IEEE Commun. Lett., 4: 190-192. DOI: 10.1109/4234.848409

13. Versaci, M. and F.C. Morabito, 2003. Fuzzy time series approach for disruption prediction in Tokamak reactors. IEEE Trans. Mag., 39: 15031506. DOI: 10.1109/TMAG.2003.810365 